\title{
Dynamic behavior of cell-free mitochondrial DNA in human saliva
}

Caroline Trumpff ${ }^{a}$, Shannon Rausser ${ }^{a}$, Rachel Haahr ${ }^{a}$, Kalpita R. Karan ${ }^{a}$, Gilles Gouspillou ${ }^{b}$, Clemens Kirschbaum ${ }^{c}$, Martin Picard ${ }^{\text {a,d,e }}$

${ }^{a}$ Department of Psychiatry, Division of Behavioral Medicine, Columbia University Irving Medical Center, New York, USA.

${ }^{b}$ Département des Sciences de l'Activité Physique, Faculté des Sciences, Université du Québec à Montréal (UQAM), Montreal, Québec, Canada.

${ }^{\mathrm{c}}$ Department of Psychology, TU Dresden, Germany.

${ }^{\mathrm{d}}$ Department of Neurology, $\mathrm{H}$. Houston Merritt Center, Columbia University Translational Neuroscience Initiative, Columbia University Irving Medical Center, New York, USA.

e New York State Psychiatric Institute, New York, USA.

Correspondence:

Martin Picard

martin.picard@columbia.edu

622 West 168th Street, $\mathrm{PH}-1540 \mathrm{~N}$

New York, NY 10032 


\begin{abstract}
Mitochondria release their genome as cell-free mitochondrial DNA (cf-mtDNA) in multiple biofluids including in the blood, where it predicts mortality and is a marker of mental and physical stress. Here we report cf-mtDNA in human saliva, an accessible biofluid used to study dynamic neuroendocrine changes. To map the natural dynamics of salivary cf-mtDNA over time, we examine cf-mtDNA and steroid hormones in a small cohort of healthy adults, and perform an intensive repeated-measures analysis of two healthy men studied at 4 daily timepoints over 5360 consecutive days ( $n=412-420$ observations). Salivary cf-mtDNA exhibits a robust awakening response reaching up to two orders of magnitude 30-45 minutes after awakening, varies from day-to-day, and moderately correlates with the cortisol awakening response. Moreover, we find no evidence that salivary cf-mtDNA has pro-inflammatory effects. The dynamic behavior of salivary cf-mtDNA opens the door to non-invasive studies examining the relevance of mtDNA signaling on human health.
\end{abstract}

Keywords: cell-free mitochondrial DNA, saliva, awakening response, steroid hormones.

\title{
Significance statement
}

Mitochondria play a key role in the pathophysiology of many human diseases, but researchers currently lack tools to reliably assess mitochondrial function and signaling at scale. Circulating cell-free mitochondrial DNA (cf-mtDNA) in blood is a marker of mental and physical stress that predicts mortality, but it is not easily accessible in large studies. This study reports the existence of cf-mtDNA in human saliva, which exhibits a dynamic behavior, including a robust awakening response and within-person day-to-day variation. We describe a robust measurement approach and document weak associations of cf-mtDNA with other mitochondria-derived steroid hormones, suggesting new avenues to examine the neuroendocrine significance of mitochondrial signaling to human health. 


\section{A. Introduction}

The maintenance of human health requires dynamic variations in key physiological systems (1). One well-described example of dynamic regulation is the hypothalamic-adrenal-pituitary (HPA) axis, which leads to cortisol release. Cortisol is not only released acutely in response to psychosocial stress $(2,3)$, but also exhibits a stereotypical diurnal pattern of variation where it rapidly increases during the first 30-45 min after awakening - a phenomenon termed the cortisol awakening response (CAR) (4). The CAR is believed to sustain the metabolic activation associated with the morning-related rise in body temperature, increased heart rate, cognitive processes, and increased energy expenditure as the organism transitions from sleep to wakefulness $(5,6)$. Diurnal variation also applies more broadly to sex hormones (7), circulating immune cell composition (8-10), metabolites (11-14), and even organ size (15), illustrating the pervasiveness of dynamic variation in human physiology.

Dynamic, diurnal cortisol changes are readily detectable in saliva, an accessible biofluid widely used in clinical and epidemiological studies, including in special populations (e.g., children), where biospecimen collection is limited to non-invasive measures. Studies using salivary cortisol have demonstrated that an abnormal CAR is linked to cardiovascular, autoimmune and psychiatric diseases (16). However, cortisol alone is not sufficient to fully explain or predict the diversity of adverse health outcomes, and one emerging contributor to psychobiological processes are the energy-producing organelle mitochondria. Mitochondria are both targets and mediators of the stress response (17), and can influence broad aspects of physiology (18), as well as development (19) and even lifespan in animal models $(20,21)$. But in human studies, we currently lack tools to track changes in mitochondrial biology from non-invasive biofluids such as saliva.

An emerging biomarker of mitochondrial stress and signaling is the circulating mitochondrial genome, which is released as cell-free mitochondrial DNA (cf-mtDNA) (22). In the blood, measuring cf-mtDNA cross-sectionally (one single collection) showed that elevated cf-mtDNA levels are associated with physical trauma (23), psychopathology $(24,25)$, infectious conditions (26), and aging (27). In the absence of dynamic time-course data, these findings led to the idea that cf-mtDNA is a relatively stable marker of disease. But subsequent studies showed that blood cf-mtDNA is rapidly inducible within 5-30 minutes after psychological stress $(28,29)$, and that within-person cf-mtDNA levels may change several-fold from week-to-week or month-to-month (22), thus challenging the notion that cf-mtDNA is a stable state biomarker. Moreover, cf-mtDNA has been detected not only in blood but also in other biofluids, including urine (30) and cerebrospinal fluid (31), indicating that cfmtDNA may ubiquitously exist in multiple biofluids. 
Together, the emerging role of cf-mtDNA in relation to health and disease states, the stressinducibility and within-person variation in blood cf-mtDNA, and its existence in multiple biofluids, led us to hypothesize that cf-mtDNA may share some dynamic properties with other neuroendocrine factors, such as cortisol, and that it may be detectable in saliva. Here we examine this hypothesis, with a focus on examining the existence and dynamic properties of salivary cf-mtDNA.

\section{B. Results}

1. cf-mtDNA is detectable in saliva and is independent of cellular content

To determine if cf-mtDNA is detectable in saliva collected using standard salivary cortisol procedures (32), saliva was collected using salivettes from a small discovery cohort of healthy adults ( $n=64$ samples from 4 women and 3 men, aged 21-72 years) at specific times of the day: at awakening, $+30 \mathrm{~min}$, and $+45 \mathrm{~min}$ after awakening. Saliva was extracted using standard procedures for cortisol, followed by an additional centrifugation step $(2,000 \mathrm{~g} \times 10 \mathrm{~min})$ to eliminate any potential contaminating cells or debris that would influence DNA levels (Fig S1A). The resulting cell-free saliva was then lysed to release cell-free DNA (cf-DNA), which was quantified by quantitative PCR (qPCR). To increase the robustness of our cf-DNA quantification, we simultaneously targeted two mitochondrial (mtDNA) and two nuclear (nDNA) amplicons (Fig S2A), as in previous work (29). As previously observed in other human biofluids, we detected substantial amounts of cf-mtDNA in human saliva. Moreover, salivary cf-mtDNA levels were within the physiological range of plasma cfmtDNA levels detected using the same methodology in an unrelated set of reference control samples (Fig 1B).

Saliva contains a large number of buccal epithelial cells and leukocytes (33), which could theoretically actively or passively release mtDNA during isolation. To test whether cellular contamination may represent a source of cf-mtDNA, we verified whether cf-mtDNA was influenced by the cellular content of saliva by quantifying the number of cells in each collected saliva sample (Fig S1B). The number of cells quantified by qPCR did not correlate with cf-mtDNA levels $\left(r^{2}=0.006\right.$, N.S.) (Fig S1C), thus ruling out cellular content as a substantial source of salivary cf-mtDNA.

\section{Sedimentation characteristics of salivary cf-mtDNA and collection methods}

As an additional test of specificity, we examined the sedimentation characteristics of salivary cfmtDNA. In blood, cf-mtDNA exists in different forms, including encapsulated within whole mitochondria, within extracellular vesicles, or as free floating DNA (22). If cf-mtDNA were contained 
in whole mitochondria, large extracellular vesicles, or apoptotic bodies, it would sediment and be depleted during high speed centrifugation (18,000g x $15 \mathrm{~min}$ ) (34). On the other hand, small vesicles or free-floating naked forms of cf-mtDNA would not. In our saliva samples, high-speed centrifugation decreased cf-mtDNA levels by only $17 \%$ on average (small effect sizes, Cohen's d=0.13-0.18, Fig S1E). Thus, less than one-fifth of saliva cf-mtDNA is contained in large vesicular structures (e.g., whole mitochondria or extracellular vesicles) whereas $>80 \%$ is either naked or contained in very small vesicular structures. These results using the first mtDNA amplicon (ND1) were confirmed with a second mtDNA amplicon (COX1), which showed high concordance (Fig S2B-D). Therefore, the remainder of our analyses use the average of both amplicons as a stable estimate of total salivary cf-mtDNA.

For typical salivary hormone analyses, saliva is collected either with salivettes (as above) or by passive drool. To evaluate the impact of the saliva collection method on cf-mtDNA levels, we compared cf-mtDNA and cf-nDNA levels from saliva collected using both methods. Participants collected saliva using both methods in a randomized, cross-over order. We observed that thawed passive drool samples tended to agglutinate, and they contained 93\% less cf-mtDNA than salivettederived saliva (Fig S3A-B). cf-mtDNA levels measured by both methods were weakly correlated $\left(r^{2}=0.05-0.10\right)$. Moreover, compared to passive drool where cf-mtDNA values were narrowly distributed near the lower limit of detection, salivette-derived saliva from the same individuals showed a higher dynamic range, suggesting that the salivette approach is technically superior (Fig S3C-D). Finally, we confirmed that freezing salivettes at -20 or $-80^{\circ} \mathrm{C}$ after collection, which is often required for long-term storage in some studies, did not significantly alter cf-mtDNA levels (Fig S3EF). Thus, the standard method of saliva collection, storage, and processing used for cortisol measurements are also appropriate to quantify cf-mtDNA. Having demonstrated that cf-mtDNA is i) abundant in human saliva, ii) independent of cellular content, and iii) robust to storage and handling conditions, provided the basis to examine cf-mtDNA dynamics.

\section{Saliva cf-mtDNA exhibits an awakening response}

To establish whether salivary cf-mtDNA exhibits an awakening response, we collected saliva at three timepoints recommended to quantify the CAR: awakening, +30 and +45 min (4). As expected, we observed relatively consistent CARs across individuals, with cortisol increasing on average from 4.80 to $10.99 \mathrm{mMol}$ from awakening to peak $(+129.06 \%, \mathrm{P}<0.0001, \mathrm{n}=15$ days, from 4 individuals, Fig 1C). A positive CAR was observed on $93 \%$ (awakening to $+30 \mathrm{~min}$ ) and $73 \%$ (awakening to +45 min) of days (Fig 1D), confirming participant compliance and the adequacy of the saliva sampling 
method. Awakening responses for other steroid hormones including cortisone, testosterone, progesterone, dehydroepiandrosterone (DHEA), and estradiol are shown in Fig S4.

Quantifying cf-mtDNA at the same timepoints revealed that salivary cf-mtDNA also exhibited a robust awakening response. On average in this small sample, cf-mtDNA increased by 2.69-fold from 0 to $+30 \mathrm{~min}(p<0.05)$ and remained elevated at $+45 \mathrm{~min}$ (Fig 1E, individual trajectories are shown in Fig 1G-M). The highest cf-mtDNA awakening response (cf-mtDNA-AR) was a 83.5-fold increase (from 915 to 76,525 A.U, 31-year-old woman, Fig. 1H). Similar to cortisol, a positive cf-mtDNA-AR was detected on $85 \%$ of days, demonstrating a consistent cf-mtDNA-AR similar in dynamics to the CAR (Fig 1F). Self-reported compliance with the timing of collection immediately at awakening was associated with the magnitude of the cf-mtDNA-AR.

We also noted that the variability in salivary cf-mtDNA levels between participants was substantial, with coefficient of variations (C.V.) ranging from $102-134 \%$ on each of the three morning time points. Moreover, in two male participants important differences in cf-mtDNA-AR were observed between two occasions of testing separated by $\sim 1$ year $(\mathbf{F i g} 1 \mathbf{L}, \mathbf{M})$, indicating potentially meaningful within-person variation in salivary cf-mtDNA dynamics over time.

\section{Within-person variation in saliva cf-mtDNA}

Understanding how salivary cf-mtDNA varies day-to-day and over time is paramount to establish its usability as a biomarker. Therefore, rather than examining cf-mtDNA in a larger cohort that would likely confirm the above results without providing additional information about the temporal dynamics of within-person variation, we performed an intensive repeated-sampling, multi-week protocol in two highly compliant participants: Participant A (34-year-old male, author M.P.); and Participant B (35year-old male, author G.G.). Using the same timepoints as above (awakening, +30 and $+45 \mathrm{~min}$ ) as well as a bedtime timepoint (bedtime) to examine potential diurnal patterns, we quantified cf-mtDNA in parallel with a salivary steroid hormone panel daily for $\sim 2$ months.

Participant A was studied over 53 consecutive days ( $n=212$ observations, 8 missing time points) (Fig 2A). Consistent with the results above and with the literature, a positive CAR was detected on $80 \%$ of days ( $p<0.0001+30 \mathrm{~min}, p<0.01+45 \mathrm{~min}$ ), with an average cortisol increase of $30 \%(5.6$ to $7.6 \mathrm{mMol}$ ), and a daytime decline from morning peak to bedtime was observed on $98 \%$ of days $(p<0.0001$, Fig 2B, D). The awakening response patterns for other steroid hormones are shown in (Fig S5). 
Across the 53 days, Participant A exhibited a robust cf-mtDNA-AR, where salivary cf-mtDNA increased on average 17.2-fold from awakening to $+45 \mathrm{~min}(P=0.0004)$ (Fig 2C, E). This diurnal cfmtDNA pattern cannot be driven by cellular contamination because salivary cellular content follows an opposite diurnal pattern; while cf-mtDNA increases after awakening, salivary cell count decreases 2.1-fold from awakening to $+30 / 45 \mathrm{~min}$ (Fig S1D). The maximal detected cf-mtDNA-AR (Day 35) was an 892-fold elevation over the first $45 \mathrm{~min}$ after awakening. These observations are in line with the data in Figure 2, albeit of larger overall magnitude, and also of substantially greater magnitude than what is observed for typical hormones such as cortisol. These results also revealed large natural day-to-day variation $(112 \% \mathrm{CV})$, calling for a similar repeated-measurements design in another participant.

Participant $B$ was studied over 60 consecutive days ( $n=220$ observations, 20 missing time points) (Fig 3A). A positive CAR was found on $66 \%$ of the days ( $p=0.021$ at $+30, p=0.045$ at +45 ), with an average magnitude of $+90 \%$ (6.4 to $12.2 \mathrm{mMol})$, and a daytime decline from morning peak to bedtime was observed on $100 \%$ of the days $(p<0.0001)$ (Fig 3B, D). See Fig S5G-K for other steroid hormones awakening patterns in Participant B.

In contrast to the normal CAR profile, the average salivary cf-mtDNA-AR pattern in Participant B was not consistent, showing an average $5-8 \%$ decline from awakening to $+30 / 45 \mathrm{~min}$ (Fig $3 E$ ). Some days exhibited a robust awakening response reaching up to 14.59-fold (Fig 3C). A cf-mtDNA morning peak to bedtime decline was observed on $72 \%$ of the days $(p=0.0003)$ (Fig $3 E$ ). Because the cf-mtDNA patterns of participant $B$ differed from the ones of the cohort and of participant $A$, all Participant B samples were re-assayed, which validated these results and showed high internal validity for the salivary cf-mtDNA assay $\left(r^{2}=0.84, p<0.0001\right.$, Fig S6). In this participant also, we observed substantial day-to-day variation in average salivary cf-mtDNA levels (50\% CV).

\section{Associations of saliva cf-mtDNA dynamics and steroid hormones}

Performing repeated-measures in the same participants makes possible to identify specific periods of time that deviate substantially from that individual's baseline or typical pattern (35), which provides a unique scientific opportunity for discovery not available in cross-sectional designs. In Participant A, we identified a 4-day period (days 27-30) where absolute cf-mtDNA levels were abnormally low (97\% lower than normal, $p=0.014)$ and the cf-mtDNA-AR was strikingly absent. On 
those days, cortisol levels also were abnormal, marked by a complete absence of CAR (Fig S7), which is unusual for this participant.

This finding prompted us to examine the correlation between salivary cf-mtDNA and cortisol. Across the 212 observations for participant $A$, the cortisol and cf-mtDNA awakening responses were moderately positively correlated $\left(r^{2}=0.19, p=0.0015\right)$ (Fig S8A). While these results were not replicated in participant $B\left(r^{2}=0.00, p=0.28\right)$, a similar effect size was found for this association in the participant cohort $\left(r^{2}=0.17, p=0.14\right)$ (Fig S8B-C). Together, this suggested a potential association between cortisol and cf-mtDNA dynamics, consistent with previous work $(24,29)$. Moreover, visual inspection of the cf-mtDNA and steroid hormone patterns showed that during the flat period where cf-mtDNA levels were abnormally low, progesterone levels were abnormally elevated by 1.38 -fold above average $(p<0.0001)$.

To explore the cortisol/cf-mtDNA association in Participant B in a hypothesis-driven manner, we isolated days with a robust CAR (+2-fold or higher from awakening to $+30 \min , \mathrm{n}=18$ ) and days without a CAR ( $n=12)$, but did not find a significant difference in the cf-mtDNA-AR between those periods (Fig S9). We note that on average, the steroid hormone patterns differed between both repeat participants. Compared to Participant A, Participant B showed a 1.3-fold higher average cortisol levels $(p<0.03)$, and higher daily variability for cortisol (2.6-fold), corticosterone (5-fold), and progesterone (20-fold) (Fig S10). During the study period, Participant B reported undergoing a regimented marathon running training program, completed a marathon on Day 27 (arrow in Fig 3A), and reported several objective major life stressors. Altogether, this suggests that the two participants may not be directly comparable, highlighting with high confidence the existence of inter-individual variation in salivary steroid hormones and cf-mtDNA dynamics.

\section{Saliva cf-mtDNA is negatively associated with IL-6 saliva levels at bedtime}

Finally, given the mixed evidence in the literature linking cf-mtDNA and pro-inflammatory states (22), we again leveraged the high-frequency, repeated-measures saliva measurements in Participant $A$ to examine if the salivary cf-mtDNA-AR was associated with the pro-inflammatory cytokine IL-6. Consistent with prior literature in plasma/serum (36) and saliva (37), salivary IL-6 exhibited a strong negative awakening response (Fig 4A). IL-6 levels were highest at awakening, decreased on average by $85-88 \%$ over the first $30-45 \mathrm{~min}(\mathrm{P}<0.0001)$, and increased by an average of 4.0 -fold from +45 min to bedtime $(P<0.0001)$. These results confirm that IL-6 and cf-mtDNA exhibit opposite awakening responses (Fig 4B). 
The opposite patterns of change between salivary cf-mtDNA and IL-6 over time leads to two conclusions. First, this provides additional evidence for the specificity of the cf-mtDNA dynamics, demonstrating that the observed changes over time are not driven by the dilution or concentration of saliva (which would change the concentration of all analytes in the same direction and in a similar magnitude). We validated this point by measuring total salivary protein concentration at multiple time points across multiple consecutive days, which confirmed that saliva concentration is relatively stable and does not vary systematically across the day, and is not correlated with cf-mtDNA (Fig S11). Second, salivary IL-6 and cf-mtDNA are modestly but significantly negatively associated $\left(r^{2}=0.08\right.$, $p<0.0001$, Fig S12A), particularly at bedtime $\left(r^{2}=0.11, p=0.016\right.$, Fig S12B). Moreover, compared to the 49 days with normal cf-mtDNA-ARs in Participant $A$, the flat 4-day period with abnormally low cf-mtDNA exhibited markedly elevated IL-6 levels (2.75-fold, $p<0.0001)$ (Fig 4C). This result corroborates the negative association between cf-mtDNA and the pro-inflammatory cytokine IL-6 (see Fig S12A).

\section{Discussion}

We have reported the existence and dynamic behavior of cf-mtDNA in human saliva. Similar to several neuroendocrine factors, such as cortisol, which exhibit natural diurnal variation and a robust awakening response, our result show that salivary cf-mtDNA exhibits a large magnitude awakening response. Our high-frequency, daily repeated-measures strategy on two different mtDNA targets also demonstrated the high day-to-day variation in cf-mtDNA, and inter-individual differences. Together, these data open the door to capturing both inter-individual differences, and inter-individual variation in salivary cf-mtDNA, to examine their associations with health- and disease-related outcomes.

Biologically, it is fascinating to consider that mitochondria are not only the major source of cellular energy, but also contribute to the biosynthesis of several neuroendocrine factors. Indeed, cortisol, as well as all steroid hormones - mineralocorticoids, estrogens, androgens - are enzymatically produced within the mitochondrial matrix (38). Cortisol is made in mitochondria of the adrenal gland zona fasciculata, testosterone is made in Leydig cells mitochondria within the testes, and estrogen is made in mitochondria within the ovaries (38). Naturally, the circulating mitochondrial genome (i.e., cfmtDNA) is also derived from mitochondria, although its exact tissue(s) or cell(s) of origin remains to be determined. Moreover, other uniquely "mitochondrial proteins" such as the heat shock protein 60 (HSP60) are released systemically in the blood, and may be elevated in relation to psychosocial stress (39) and cardiovascular disease (40). In our data, the generally small correlations found 
between cortisol and cf-mtDNA make it unlikely that they would be co-released from the same mitochondria. Rather, we speculate that distinct mitochondria-derived signals are released by different source mitochondria, to fulfill regulatory roles within the organism (41). The role that cfmtDNA plays within the organism largely remains to be determined.

A major current knowledge gap relates to the mechanism responsible for the rapid release and dynamics of cf-mtDNA. Studies in blood showed that cf-mtDNA can change by 2-3-fold in serum and plasma over 5-30 minutes $(28,29)$, and that cf-mtDNA levels measured at one timepoint can vary up to an order of magnitude when measured again within weeks or months (22). These data suggested that blood cf-mtDNA is not a stable trait of the individual, but may present a dynamic behavior. Our data confirms this assertion, and shows that salivary cf-mtDNA can increase by over 100-fold over periods of 15-30 minutes, establishing its highly dynamic nature. In comparison, salivary cortisol increases by 2-to-5 fold within 20-30 minutes post-stress, or more moderately after awakening, and decreases with similar kinetics (3). Based on these data, cf-mtDNA and cortisol appear to have similar kinetics but differ mainly in the magnitude of their variation, although more systematic and higher temporal resolution data are necessary to confirm this point. In general, our measurements show that the salivary cf-mtDNA-AR is likely $\sim 10-100$ fold larger than the average salivary CAR. Mechanistically, we found that cortisol and cf-mtDNA were only weakly correlated, suggesting they are either unlikely causally linked to one another, or that cortisol in itself may contribute to increases in cf-mtDNA, but is not sufficient to do so on its own.

Technically, our confidence that cf-mtDNA is a robust and specific biological entity is enhanced by three observations. First, cf-mtDNA is independent of the number of cells in saliva, which makes it unlikely that salivary cf-mtDNA is mainly released locally by buccal epithelial cells or leukocytes. This also confirms that cf-mtDNA is not technically confounded by cellular content or other factors related to sample preparation, such as the salivette collection method. Second, the vast majority of cf-mtDNA at different time points exists in non-sedimentable form (i.e., not precipitated by high centrifugal force). This indicates that cf-mtDNA exists in a biological form of transport that is either membrane free, such as naked DNA, or encapsulated by small microvesicles, and that this is mostly consistent across all timepoints analyzed. Third, analyses of total protein and IL-6 concentrations both confirm that the large observed variations in cf-mtDNA cannot be attributed to changes in overall saliva concentration. More research is required to determine the exact form of transport and origin of salivary cf-mtDNA (22) and whether there is only one major form of salivary cf-mtDNA, or multiple 
forms. Further research is also needed to establish whether salivary cf-mtDNA levels reflect cf-mtDNA in the blood and other biofluids.

Methodologically, using saliva to study cf-mtDNA levels and dynamics may have several advantages over more invasive measures: i) saliva samples can be easily collected to study cfmtDNA behavior in vulnerable populations including children (42), ii) accessing blood or cerebrospinal fluid may induce stress and/or pain, which could influence cf-mtDNA levels, and iii) existing epidemiological studies and biobanks with stored saliva samples are amenable to cf-mtDNA measurements after an additional centrifugation step to eliminate potential cellular contamination. Moreover, since salivary cf-mtDNA is robust to freezing effects, saliva samples may be conveniently collected and stored frozen at home, which can greatly enhance the ecological validity of future mitochondrial psychobiology studies.

In summary, our findings demonstrate the existence of cf-mtDNA in human saliva and provide a method to quantify its dynamic behavior, including its robust awakening response and diurnal variation. This finding greatly expands our knowledge of cf-mtDNA in humans, and defines a broad range of dynamics in humans. Thus, this work provides a basis for larger well-controlled studies required to understand the significance of salivary cf-mtDNA in relation to key health-related factors, including behaviors and psychosocial stress, as well as mental and physical health states.

\section{Method}

\section{Participants}

All procedures for this study were approved by New York State Psychiatric Institute (Protocol \# 7748). All participants provided written informed consent for their participation and publication of the results. Healthy adults between 18 and 100 years were eligible for inclusion. Exclusion criteria included current diagnosis of cancer or chronic inflammatory disorders, involvement in other clinical trials, and pregnancy. Participants were recruited via flyers posted in the Columbia University Irving Medical Center (CUIMC) community and using a snowball recruitment strategy. The discovery cohort included 7 individuals (4 women, 3 men), aged 21-72 years old. To circumvent the high participant burden and potential cost associated with collecting multiple daily samples across multiple consecutive weeks, repeated daily measures were collected across 53 and 60 days from two healthy Caucasian men (authors M.P., 34 years old, and G.G., 35 years old) to assess within-person variability of salivary cf-mtDNA. 


\section{Saliva collection}

Saliva was collected at four time points: in the mornings i) immediately upon awakening, ii) 30 minutes after waking up, iii) 45 minutes after waking up, and iv) at bedtime. A total of 1-2 ml of saliva was collected at each sample timepoint using a Starstedt Salivette (Cat\# 51.1534.500) following the recommended procedure from the Biomarker Network (32). Participants were instructed to place the cotton swab in their mouth (middle of the tongue) for 2-5 minutes, not bite down on the cotton roll, and to avoid contact with the cheeks. Participants then placed the cotton swab back into the Salivette tube and immediately stored the sample in the freezer to preserve analytes (e.g., cf-mtDNA, steroid hormones, IL-6). In the morning, participants avoided brushing their teeth until after the third (+45 min) sample, and the night time sample was collected prior to teeth brushing at bedtime. Participants were instructed not to eat before the +45 min timepoint, and were instructed not to drink water or other fluids 10 minutes before each sample collection. All participants stored salivettes in their freezer $\left(-20^{\circ} \mathrm{C}\right)$ before either transporting or shipping samples to the laboratory.

\section{Salivette processing and storage}

Following standard recommended procedures for cortisol, salivettes were thawed and subsequently centrifuged at $1,000 \times \mathrm{g}$ for 5 minutes in a refrigerated $4^{\circ} \mathrm{C}$ centrifuge. From the top fraction of saliva, $500 \mathrm{ul}$ was collected and stored at $-80^{\circ} \mathrm{C}$ for steroid hormone analysis. 200 ul was transferred to a $1.5 \mathrm{ml}$ Eppendorf tube and stored at $-80^{\circ} \mathrm{C}$ for cf-DNA analysis. The remaining saliva was removed, leaving behind $\sim 50$ ul to resuspend total salivary cells (leukocytes and buccal epithelial cells) sedimented at the bottom of the salivette. Cells were collected and transferred to a fresh tube, centrifuged at $2,000 \times \mathrm{g}$ for 2 minutes at $4^{\circ} \mathrm{C}$, and the supernatant carefully aspirated, leaving behind a clean cell pellet. This cell pellet was used to quantify the total salivary cellular content using the nuclear DNA amplicon B2M.

\section{Quantification of cf-mtDNA}

To ensure the absence of cell and cell debris, each saliva sample was further centrifuged at 2,000 $\mathrm{xg}$ for 10 minutes at $4^{\circ} \mathrm{C}$ prior to processing. To avoid bias in mtDNA content that may occur during DNA isolation using column-based methods (43), DNA was extracted using an isolation-free lysis method, adapted from (44). 20 ul of saliva was added to 180 ul of lysis buffer (500mM Tris HCL, $1 \%$ Tween $20, \mathrm{dH}_{2} 0$, and $20 \mathrm{ug} / \mathrm{ml}$ proteinase $\mathrm{K}$ ), for a dilution of 1:10. Lysis was performed for 10 hours at $55^{\circ} \mathrm{C}$, followed by inactivation at $95^{\circ} \mathrm{C}$ for 10 minutes. This lysis-based method was adapted from a method originally developed for single-cell analysis (45), which allows the detection of small 
amounts of genomic material without DNA isolation bias inherent to standard column-based DNA isolation techniques $(46,47)$.

Eight ul of the lysed saliva was used directly as template DNA for qPCR. qPCR reactions were run in triplicates using a liquid handling station (ep-Motion5073, Eppendorf) in 384 well qPCR plates. Duplex qPCR reactions with Taqman chemistry were used to simultaneously quantify mtDNA and nDNA amplicons in the same reactions. Master Mix ${ }_{1}$ for ND1 (mtDNA) and B2M (nDNA) included: Taqman Universal Master mix fast (life technologies \#4444964), $300 \mathrm{nM}$ of primers and $100 \mathrm{nM}$ probe (ND1-Fwd: GAGCGATGGTGAGAGCTAAGGT, ND1-Rev: CCCTAAAACCCGCCACATCT, Probe:HEX-CCATCACCCTCTACATCACCGCCC-3IABkFQ.B2M-

Fwd:CCAGCAGAGAA TGGAAAGTCAA,B2M-Rev: TCTCTCTCCATTCTTCAGTAAGTCAACT, Probe:FAM- ATGTGTCTGGGTTTCATCCATCCGACA-3IABkFQ). Master Mix 2 for COX1 (mtDNA) and RnaseP (nDNA) included: TaqMan Universal Master Mix fast, $300 \mathrm{nM}$ of primers and $100 \mathrm{nM}$ probe (COX1-Fwd: CTAGCAGGTGTCTCCTCTATCT, GAGAAGTAGGACTGCTGTGATTAG, Probe:

TGCCATAACCCAATACCAAACGCC-3IABkFQ. AGATTTGGACCTGCGAGCG, RnaseP-Rev: GAGCGGCTGTCTCCACAAGT, Probe: FAMTTCTGACCTGAAGGCTCTGCGCG-3IABkFQ. The samples were then cycled in a QuantStudio 7 flex qPCR instrument (Applied Biosystems Cat\# 4485701) at $50^{\circ} \mathrm{C}$ for $2 \mathrm{~min}, 95^{\circ} \mathrm{C}$ for $20 \mathrm{sec}, 95^{\circ} \mathrm{C}$ for $1 \mathrm{~min}, 60^{\circ} \mathrm{C}$ for $20 \mathrm{sec}$, for 40 cycles. Reaction volumes were $20 \mathrm{ul}$. To ensure comparable Ct values across plates and assays, thresholds for fluorescence detection for both mitochondrial and nuclear amplicons were set to 0.08 .

cf-DNA was calculated by linearizing the log-based Ct values $\left(2^{\mathrm{Ct}}\right)$ into linear space to obtain quantitative estimates of cf-mtDNA levels in arbitrary units. Because higher cf-mtDNA content is represented by lower $\mathrm{Ct}$ values (more template mtDNA copies amplified earlier), the inverse of the linear values were obtained and divided by an arbitrary threshold $\left(1^{-12}\right)$ that represents the lower detection limit for mtDNA on our instrument (corresponding $\mathrm{Ct} \sim 36$ ). Undetectable cf-DNA values were replaced by 1 . The final cf-mtDNA values were obtained from the average of two mtDNA genes (ND1 and COX1) and cf-nDNA was obtained from the average of two single-copy nDNA genes (B2M and RNaseP).

\section{Steroid hormones}

Salivary steroid hormones, including cortisol, cortisone, testosterone, progesterone, corticosterone and dehydroepiandrosterone (DHEA), were quantified using a high throughput liquid chromatography-tandem mass spectrometry (LC-MS/MS) assay as described previously (48). The 
lowest detectable limit was $0.0055 \mathrm{nmol} / \mathrm{l}$ for cortisol, $0.0019 \mathrm{ng} / \mathrm{ml}$ for cortisone, $2 \mathrm{pg} / \mathrm{ml}$ for testosterone, $2.3 \mathrm{pg} / \mathrm{ml}$ for progesterone, $5.35 \mathrm{pg} / \mathrm{ml}$ for DHEA and $0.9 \mathrm{pg} / \mathrm{ml}$ for estradiol. Values below the limit of detection were imputed as the value of the detection limit.

\section{Salivary protein concentration}

Total salivary protein concentration was measured in 44 consecutive samples using the bicinchoninic acid (BCA) protein assay (Bio-World, Cat. \#20831001-1), in a 96-well microplate, using endpoint absorbance (optical density, $562 \mathrm{~nm}$ OD) on a SpectraMax M3 (Molecular Devices). Saliva samples were diluted 1:10 with nuclease-free water to ensure that all samples were within the assay's dynamic range, and measured in duplicates. Absolute protein concentration in each sample was determined in a single batch from a standard curve with bovine serum albumin (BSA), adjusting for the dilution factor. Final values are expressed in $\mathrm{mg}$ protein per $\mathrm{ml}$ of saliva, and ranged from 0.8$4.8 \mathrm{mg} / \mathrm{ml}($ median $=1.6 \mathrm{mg} / \mathrm{ml})$.

\section{IL-6 quantification}

Salivary IL-6 concentration was measured by ELISA using the Human IL-6 Quantikine HS ELISA kit (R\&D Systems, Cat\# HS600C) following the manufacturer's instructions. After an optimization step, all saliva samples were diluted 1:4 with the diluent, ensuring that all samples were within the assay's dynamic range. The sample absorbance was determined in microplates on a SpectraMax M3 (Molecular Devices) at $450 \mathrm{~nm}$ and $570 \mathrm{~nm}$. A standard curve was used to extrapolate unknown saliva IL-6, multiplied by the dilution factor. Batch-to-batch variations were mathematically corrected using a reference serum sample with known IL-6 concentration included systematically on each IL-6 array. The final IL-6 concentrations are expressed in $\mathrm{pg} / \mathrm{ml}$, with a range of $0.1-92 \mathrm{pg} / \mathrm{ml}$ (median $=6 \mathrm{pg} / \mathrm{ml})$.

\section{Statistical analysis}

For both salivary cortisol and cf-mtDNA, Chi-square tests were used to compare proportions of positive and negative awakening response, and proportions of positive and negative slope from morning peak values to bedtime in levels compared to proportions expected by chance (50:50). Bilateral unpaired t-tests were used to compare cf-mtDNA levels obtained using passive drool and salivette, as well as samples frozen and refrigerated. Variation in cf-mtDNA levels and steroid hormones during the day was assessed using repeated measures One way ANOVA. Spearman's $r$ ( $r$ ) and/or simple linear regression were used to assess the strength of the associations between cfmtDNA, steroid hormones, and IL-6. Between- and within-person variations were characterized using 
bioRxiv preprint doi: https://doi.org/10.1101/2021.09.15.460321; this version posted September 15, 2021. The copyright holder for this preprint (which was not certified by peer review) is the author/funder, who has granted bioRxiv a license to display the preprint in perpetuity. It is made available under aCC-BY-NC-ND 4.0 International license.

coefficients of variation (C.V.). DHEA in participant B had more than $50 \%$ missing values, so DHEA values were excluded from the analysis. To characterize typical diurnal response, values above 1.5 $x(I Q R)$ to the third quartile were removed (in participant $B$ ). Statistical analyses were performed with Prism 8 (GraphPad, CA), R version 4.0.2 and RStudio version 1.3.1056. Statistical significance was set at $p<0.05$. 


\section{Author contributions}

$\mathrm{CT}, \mathrm{SR}$, and MP designed the research. SR recruited participants, coordinated data collection, and performed validation experiments. SR, KRK, and RH processed samples and collected data. SR performed the cf-mtDNA and IL-6 measurements, RH the BCA measurements, and CK the steroid hormone measurements. CT and SR analyzed and interpreted data. CT and MP drafted the manuscript. All authors reviewed and commented on the final version of the manuscript.

\section{Acknowledgements}

We would like to acknowledge Celina Porcaro for her work on an initial phase of this project, and Brett Kaufman for useful comments on these data.

\section{Funding}

This work was supported by the Wharton Fund and $\mathrm{NIH}$ grant $\mathrm{MH} 123927$.

\section{Conflict of interest}

The authors declare no competing interest. 


\section{References}

1. P. Sterling, What Is Health?: Allostasis and the Evolution of Human Design (MIT Press, 2020).

2. A. Caplin, F. Chen, M. Beauchamp, E. Puterman, The effects of exercise intensity on the cortisol response to a subsequent acute psychosocial stressor.

Psychoneuroendocrinology, 105336 (2021).

3. P. Foley, C. Kirschbaum, Human hypothalamus-pituitary-adrenal axis responses to acute psychosocial stress in laboratory settings. Neuroscience \& Biobehavioral Reviews 35, 91-96 (2010).

4. T. Stalder et al., Assessment of the cortisol awakening response: Expert consensus guidelines. Psychoneuroendocrinology 63, 414-432 (2016).

5. W. Schlotz, J. Hellhammer, P. Schulz, A. A. Stone, Perceived work overload and chronic worrying predict weekend-weekday differences in the cortisol awakening response. Psychosomatic medicine 66, 207-214 (2004).

6. N. Rohleder, S. E. Beulen, E. Chen, J. M. Wolf, C. Kirschbaum, Stress on the dance floor: the cortisol stress response to social-evaluative threat in competitive ballroom dancers. Personality and Social Psychology Bulletin 33, 69-84 (2007).

7. D. J. Brambilla, A. M. Matsumoto, A. B. Araujo, J. B. McKinlay, The effect of diurnal variation on clinical measurement of serum testosterone and other sex hormone levels in men. The Journal of Clinical Endocrinology \& Metabolism 94, 907-913 (2009).

8. K. Ackermann et al., Diurnal rhythms in blood cell populations and the effect of acute sleep deprivation in healthy young men. Sleep 35, 933-940 (2012).

9. C. Scheiermann et al., Adrenergic nerves govern circadian leukocyte recruitment to tissues. Immunity 37, 290-301 (2012).

10. J. Born, T. Lange, K. Hansen, M. Mölle, H.-L. Fehm, Effects of sleep and circadian rhythm on human circulating immune cells. The Journal of Immunology 158, 4454-4464 (1997).

11. J. E. Ang et al., Identification of human plasma metabolites exhibiting time-of-day variation using an untargeted liquid chromatography-mass spectrometry metabolomic approach. Chronobiology international 29, 868-881 (2012).

12. E. C.-P. Chua et al., Extensive diversity in circadian regulation of plasma lipids and evidence for different circadian metabolic phenotypes in humans. Proceedings of the National Academy of Sciences 110, 14468-14473 (2013). 
13. R. Dallmann, A. U. Viola, L. Tarokh, C. Cajochen, S. A. Brown, The human circadian metabolome. Proceedings of the National Academy of Sciences 109, 2625-2629 (2012).

14. T. Kasukawa et al., Human blood metabolite timetable indicates internal body time. Proceedings of the National Academy of Sciences 109, 15036-15041 (2012).

15. Y. Liu et al., Genetic architecture of 11 organ traits derived from abdominal MRI using deep learning. Elife 10, e65554 (2021).

16. B. Kudielka, C. Kirschbaum, Awakening cortisol responses are influenced by health status and awakening time but not by menstrual cycle phase.

Psychoneuroendocrinology 28, 35-47 (2003).

17. M. Picard, B. S. McEwen, Psychological Stress and Mitochondria: A Systematic Review. Psychosom Med 80, 141-153 (2018).

18. M. Picard et al., Mitochondrial functions modulate neuroendocrine, metabolic, inflammatory, and transcriptional responses to acute psychological stress. Proceedings of the National Academy of Sciences 112, E6614-E6623 (2015).

19. A. Quintana, S. E. Kruse, R. P. Kapur, E. Sanz, R. D. Palmiter, Complex I deficiency due to loss of Ndufs4 in the brain results in progressive encephalopathy resembling Leigh syndrome. Proceedings of the National Academy of Sciences 107, 10996-11001 (2010).

20. A. Latorre-Pellicer et al., Mitochondrial and nuclear DNA matching shapes metabolism and healthy ageing. Nature 535, 561-565 (2016).

21. M. Akbari, T. B. Kirkwood, V. A. Bohr, Mitochondria in the signaling pathways that control longevity and health span. Ageing research reviews 54, 100940 (2019).

22. C. Trumpff et al., Stress and circulating cell-free mitochondrial DNA: a systematic review of human studies, physiological considerations, and technical recommendations. Mitochondrion (2021).

23. N. Y. Lam, T. H. Rainer, R. W. Chiu, G. M. Joynt, Y. D. Lo, Plasma mitochondrial DNA concentrations after trauma. Clinical chemistry 50, 213-216 (2004).

24. D. Lindqvist et al., Increased plasma levels of circulating cell-free mitochondrial DNA in suicide attempters: associations with HPA-axis hyperactivity. Translational psychiatry $\mathbf{6}$, e971 (2016).

25. D. Lindqvist et al., Circulating cell-free mitochondrial DNA, but not leukocyte mitochondrial DNA copy number, is elevated in major depressive disorder. Neuropsychopharmacology 43, 1557-1564 (2018). 
26. K. Nakahira et al., Circulating mitochondrial DNA in patients in the ICU as a marker of mortality: derivation and validation. PLoS medicine 10, e1001577 (2013).

27. M. Pinti et al., Circulating mitochondrial DNA increases with age and is a familiar trait: Implications for "inflamm-aging". European journal of immunology 44, 1552-1562 (2014).

28. E. Hummel et al., Cell-free DNA release under psychosocial and physical stress conditions. Translational Psychiatry 8, 236 (2018).

29. C. Trumpff et al., Acute psychological stress increases serum circulating cell-free mitochondrial DNA. Psychoneuroendocrinology 106, 268-276 (2019).

30. K. Kim et al., Clinical relevance of cell-free mitochondrial DNA during the early postoperative period in kidney transplant recipients. Scientific reports 9, 1-9 (2019).

31. K. N. Varhaug et al., Increased levels of cell-free mitochondrial DNA in the cerebrospinal fluid of patients with multiple sclerosis. Mitochondrion 34, 32-35 (2017).

32. C. Kirschbaum, D. H. Hellhammer, Salivary cortisol in psychoneuroendocrine research: recent developments and applications. Psychoneuroendocrinology 19, 313-333 (1994).

33. C. Theda et al., Quantitation of the cellular content of saliva and buccal swab samples. Scientific reports 8, 1-8 (2018).

34. Z. A. A. Dache et al., Blood contains circulating cell free respiratory competent mitochondria. FASEB Journal 34, 3616-3630 (2020).

35. R. A. Poldrack et al., Long-term neural and physiological phenotyping of a single human. Nature communications 6, 1-15 (2015).

36. G. Nilsonne, M. Lekander, T. Åkerstedt, J. Axelsson, M. Ingre, Diurnal variation of circulating interleukin-6 in humans: a meta-analysis. PLoS One 11, e0165799 (2016).

37. S. Izawa, K. Miki, X. Liu, N. Ogawa, The diurnal patterns of salivary interleukin-6 and Creactive protein in healthy young adults. Brain, behavior, and immunity 27, 38-41 (2013).

38. A. Midzak, V. Papadopoulos, Adrenal mitochondria and steroidogenesis: from individual proteins to functional protein assemblies. Frontiers in endocrinology 7, 106 (2016).

39. A. Shamaei-Tousi et al., Plasma heat shock protein 60 and cardiovascular disease risk: the role of psychosocial, genetic, and biological factors. Cell stress \& chaperones 12 , 384-392 (2007).

40. A. G. Pockley et al., Circulating heat shock protein 60 is associated with early cardiovascular disease. Hypertension 36, 303-307 (2000). 
41. M. Picard, B. S. McEwen, E. Epel, C. Sandi, An energetic view of stress: Focus on mitochondria. Frontiers in neuroendocrinology (2018).

42. K. K. Ridout et al., Childhood Maltreatment, Behavioral Adjustment, and Molecular Markers of Cellular Aging in Preschool-Aged Children: A Cohort Study.

Psychoneuroendocrinology 107, 261-269 (2019).

43. S. A. Ware et al., An automated, high throughput methodology optimized for quantitative cell-free mitochondrial and nuclear DNA isolation from plasma. Journal of Biological Chemistry, jbc. RA120. 015237 (2020).

44. M. Picard et al., Mitochondrial dysfunction and lipid accumulation in the human diaphragm during mechanical ventilation. Am J Respir Crit Care Med 186, 1140-1149 (2012).

45. L. Zhou, A. Chomyn, G. Attardi, C. A. Miller, Myoclonic epilepsy and ragged red fibers (MERRF) syndrome: selective vulnerability of CNS neurons does not correlate with the level of mitochondrial tRNAlys mutation in individual neuronal isolates. Journal of Neuroscience 17, 7746-7753 (1997).

46. A. Bender et al., High levels of mitochondrial DNA deletions in substantia nigra neurons in aging and Parkinson disease. Nature genetics 38, 515-517 (2006).

47. A. Grünewald et al., Mitochondrial DNA Depletion in Respiratory Chain-Deficient P arkinson Disease Neurons. Annals of neurology 79, 366-378 (2016).

48. W. Gao, T. Stalder, C. Kirschbaum, Quantitative analysis of estradiol and six other steroid hormones in human saliva using a high throughput liquid chromatographytandem mass spectrometry assay. Talanta 143, 353-358 (2015). 
bioRxiv preprint doi: https://doi.org/10.1101/2021.09.15.460321; this version posted September 15,2021 . The copyright holder for this preprint (which was not certified by peer review) is the author/funder, who has granted bioRxiv a license to display the preprint in perpetuity. It is made available under aCC-BY-NC-ND 4.0 International license.


Figure 1. Detectable levels of cf-mtDNA and the awakening response in human saliva. (A) The goal of this study was to assess whether cf-mtDNA is detectable in saliva. (B) Distribution of cf-mtDNA (lines represent the mean) measured in saliva ( $\mathrm{n}=65$ observations in 4 women and 3 men, aged $21-72$ years old) and plasma ( $n=85$ observations measured in an independent reference cohort from healthy participants). The data was log(2) transformed prior to visualization. (C) To assess potential salivary cf-mtDNA awakening response, saliva was collected at awakening, 30 and 45 min after awakening. Cortisol awakening response in a subset of 4 participants (22 and 31 years old women, 25 and 34 years old men) studied over 3-4 consecutive days. (D) Distribution of fold change from awakening to $30 \mathrm{~min}$ (left) and awakening to $45 \mathrm{~min}$ (right) (15 assessments from a total of 4 participants) in cortisol levels. (E) Salivary cf-mtDNA values for 7 participants ( $n=65$ observations in 4 women, 3 men, aged 21-72 years old). Some participants were studied over 3-4 consecutive days and 2 were studied again one year later. (F) Distribution of fold change from awakening to 30 min (left) and awakening to 45 min (right) (20 assessments from a total of 7 participants) in cf-mtDNA levels. (G-M) Individuals profiles of cf-mtDNA awakening response. Data is shown as means \pm SEM. P-values from One-way ANOVA for repeated measures (main effect) (B,D), from chi-squared test comparing proportion of positive and negative awakening responses compared to chance (50:50) $(C, E), p<0.05^{\star}, p<0.01^{\star \star}, p<0.001^{\star \star \star}, p<0.0001^{\star \star \star *}$. 
bioRxiv preprint doi: https://doi.org/10.1101/2021.09.15.460321; this version posted September 15,2021 . The copyright holder for this preprint (which was not certified by peer review) is the author/funder, who has granted bioRxiv a license to display the preprint in perpetuity. It is made available under aCC-BY-NC-ND 4.0 International license.

Daily profiles of salivary cf-mtDNA and steroid hormones


Figure 2. Salivary cf-mtDNA diurnal variation in participant A. (A) Day-to-day variation of salivary cf-mtDNA, steroid hormones and IL-6 in a 34 year-old male participant (Participant A). (Top) Salivary cf-mtDNA collected at awakening, 30 and 45 min after awakening, and bedtime over 53 consecutive days ( $n=212$ observations). 53-day diurnal profiles for (B) cortisol and (C) cf-mtDNA. (D) Fold change in saliva cortisol from awakening to +30 , awakening to +45 and morning peak to evening values. (E) Same as in (D) for salivary cf-mtDNA. (B, C) Data is shown as means and individual datapoints. P-value from Oneway ANOVA for repeated measures (main effect). (D, E) P-value from chi-squared test comparing proportion of positive and negative awakening response compared to chance (50:50), $p<0.05^{\star}, p<0.01^{\star \star}, p<0.001^{\star \star \star}, p<0.0001^{\star \star \star}$. 
bioRxiv preprint doi: https://doi.org/10.1101/2021.09.15.460321; this version posted September 15, 2021. The copyright holder for this preprint (which was not certified by peer review) is the author/funder, who has granted bioRxiv a license to display the preprint in perpetuity. It is made available under aCC-BY-NC-ND 4 .0 International license.
Daily profiles of salivary cf-mtDNA and steroid hormones
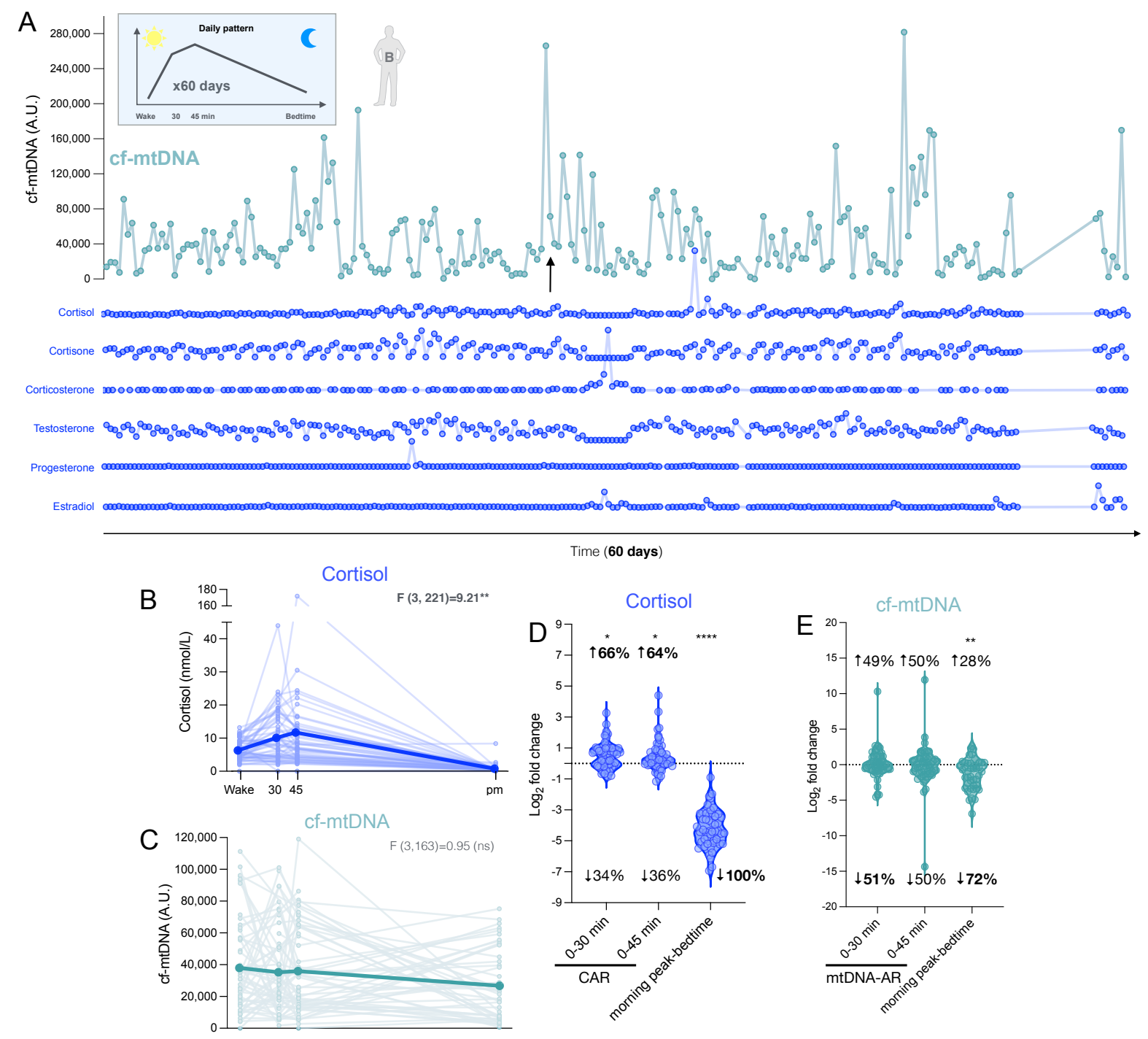

Figure 3. Salivary cf-mtDNA diurnal variation in Participant B. (A) Day-to-day variation of salivary cf-mtDNA, steroid hormones in a 35 year-old male participant (Participant B). (Top) Salivary cf-mtDNA collected at awakening, 30 and 45 min after awakening, and bedtime over 60 consecutive days ( $\mathrm{n}=220$ observations). 60-day diurnal profiles for (B) cortisol and (C) cf-mtDNA (without outliers). (D) Fold change in saliva cortisol from awakening to +30, awakening to +45 and morning peak to evening values. (E) Same as in (D) for salivary cf-mtDNA. (F) 60-day diurnal profiles for (G) cortisol and (H) cfmtDNA. Same as in D-E for (I) cortisol and ( $\mathbf{J}$ ) cf-mtDNA (extreme values removed). (B, C) Data is shown as means and individual datapoints. P-value from One-way ANOVA for repeated measures (main effect). (D, E) P-value from chi-squared test comparing proportion of positive and negative awakening response compared to chance (50:50), $\mathrm{p}<0.05^{\star}, \mathrm{p}<0.01^{\star \star}, \mathrm{p}<0.001^{\star \star \star}, \mathrm{p}<0.0001^{\star \star \star}$. 
bioRxiv preprint doi: https://doi.org/10.1101/2021.09.15.460321; this version posted September 15, 2021. The copyright holderfor this preprint

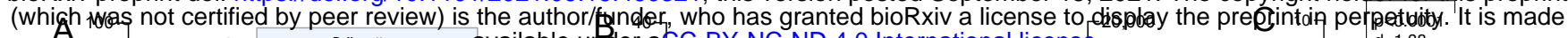
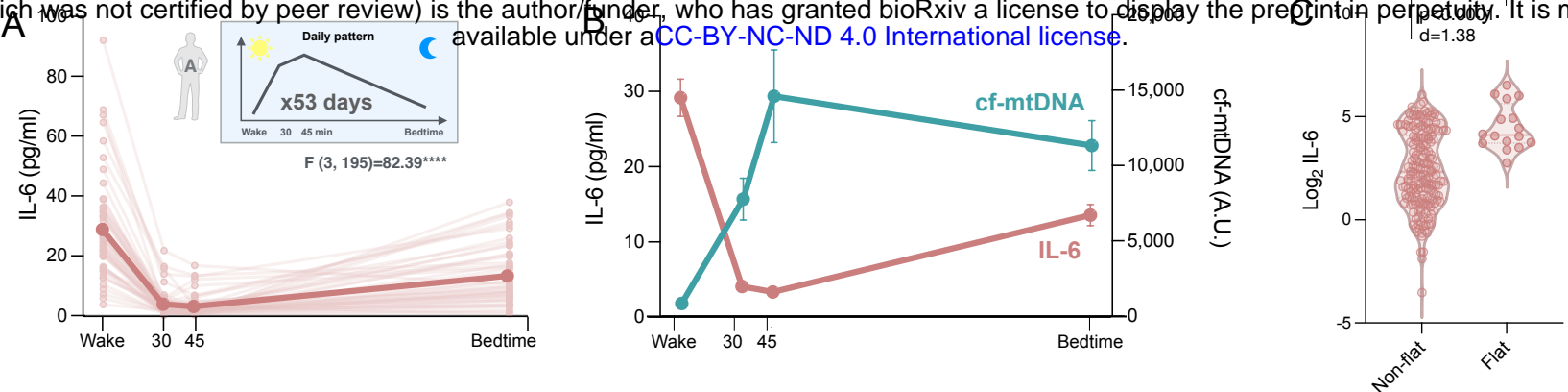

Figure 4. Salivary IL-6 and cf-mtDNA diurnal variation. (A) IL- 6 diurnal patterns in participant A with data collected over 53 days at awakening, +30 min, +45 min and evening ( $n=204$ observations). Data is shown as means and individual datapoints. P-value from One-way ANOVA for repeated measures (main effect). (B) Trajectories of salivary IL-6 and cf-mtDNA. Data is shown as means \pm SEM. (C) Comparison of IL-6 levels on normal days (non-flat) and days with unusually low cf-mtDNA levels (flat) (see figure S7). IL-6 values were $\log _{2}$ transformed prior to visualization. $\mathrm{P}$-value from unpaired t-test. $\mathrm{p}<0.0001^{\star \star \star \star}$. 